\title{
Genomics versus mtDNA for resolving stock structure in the silky shark (Carcharhinus falciformis)
}

\author{
Derek W. Kraft ${ }^{\text {Corresp., }}{ }^{1}$, Emily Conklin ${ }^{1}$, Evan Barba ${ }^{1}$, Melanie Hutchinson ${ }^{1,2}{ }^{2}$, Robert J. Toonen ${ }^{1}$, Zac H. Forsman ${ }^{1}$, \\ Brian W. Bowen ${ }^{1}$ \\ 1 Hawai'i Institute of Marine Biology, University of Hawai'i, Kaneohe, Hawaii, USA \\ ${ }^{2}$ Joint Institute of Marine and Atmospheric Research, Pacific Islands Fisheries Science Center, NOAA, University of Hawai'i, Honolulu, Hawaii, USA \\ Corresponding Author: Derek W. Kraft \\ Email address: Kraftd@hawaii.edu
}

Conservation genetic approaches for elasmobranchs have focused on regions of the mitochondrial genome or a handful of nuclear microsatellites. High-throughput sequencing offers a powerful alternative for examining population structure using many loci distributed across the nuclear and mitochondrial genomes. These single nucleotide polymorphisms are expected to provide finer scale and more accurate population level data; however, there have been few genomic studies applied to elasmobranch species. The desire to apply next-generation sequencing approaches is often tempered by the costs, which can be offset by pooling specimens prior to sequencing (pool-seq). In this study, we assess the utility of pool-seq by applying this method to the same individual silky sharks, Carcharhinus falciformis, previously surveyed with the mtDNA control region in the Atlantic and Indian Oceans. Pool-seq methods were able to recover the entire mitochondrial genome as well as thousands of nuclear markers. This volume of sequence data enabled the detection of population structure between regions of the Atlantic Ocean populations, undetected in the previous study (inter-Atlantic mitochondrial SNPS FST values comparison ranging from 0.029 to 0.135 and nuclear SNPs from 0.015 to 0.025 ). Our results reinforce the conclusion that sampling the mitochondrial control region alone may fail to detect finescale population structure, and additional sampling across the genome may increase resolution for some species. Additionally, this study shows that the costs of analyzing 4,988 loci using pool-seq methods are equivalent to the standard Sanger-sequenced markers and become less expensive when large numbers of individuals (>300) are analyzed. 


\section{Genomics versus mtDNA for Resolving Stock Structure in the Silky Shark}

\section{2 (Carcharhinus falciformis)}

3

4 Derek W. Kraft ${ }^{*}$, Emily Conklin ${ }^{1}$, Evan Barba ${ }^{1}$, Melanie Hutchinson ${ }^{1,2}$, Robert J. Toonen ${ }^{1}$, 5 Zac H. Forsman ${ }^{1}$, Brian W. Bowen ${ }^{1}$

7 'Hawai'i Institute of Marine Biology, University of Hawai'i, 46-007 Lilipuna Road, Kāne‘ohe,

8 HI 96744

9 2Joint Institute for Marine and Atmospheric Research, University of Hawai ‘i, Pacific Islands

10 Fisheries Science Center, NOAA

11

12 Corresponding Author:

13 Derek W. Kraft ${ }^{1}$

14 46-007 Lilipuna Rd, Kaneohe, HI 96744, USA

15

16 Email address: Kraftd@hawaii.edu 


\section{Abstract}

27 Conservation genetic approaches for elasmobranchs have focused on regions of the mitochondrial genome or a handful of nuclear microsatellites. High-throughput sequencing offers a powerful alternative for examining population structure using many loci distributed across the nuclear and mitochondrial genomes. These single nucleotide polymorphisms are expected to provide finer scale and more accurate population level data; however, there have been few genomic studies applied to elasmobranch species. The desire to apply next-generation sequencing approaches is often tempered by the costs, which can be offset by pooling specimens

34 prior to sequencing (pool-seq). In this study, we assess the utility of pool-seq by applying this method to the same individual silky sharks, Carcharhinus falciformis, previously surveyed with the mtDNA control region in the Atlantic and Indian Oceans. Pool-seq methods were able to recover the entire mitochondrial genome as well as thousands of nuclear markers. This volume of sequence data enabled the detection of population structure between regions of the Atlantic Ocean populations, undetected in the previous study (inter-Atlantic mitochondrial SNPs $\mathrm{F}_{\mathrm{ST}}$ values comparison ranging from 0.029 to 0.135 and nuclear SNPs from 0.015 to 0.025 ). Our

41 results reinforce the conclusion that sampling the mitochondrial control region alone may fail to 42 detect fine-scale population structure, and additional sampling across the genome may increase resolution for some species. Additionally, this study shows that the costs of analyzing 4,988 loci using pool-seq methods are equivalent to the standard Sanger-sequenced markers and become less expensive when large numbers of individuals $(>300)$ are analyzed.

\section{Introduction}


48 Many elasmobranchs around the globe have experienced devastating population declines due to

49 overfishing in both target and non-target fisheries (Musick et al., 2000; Clarke et al., 2006; F.

50 Ferretti et al., 2010; Heupel et al., 2014; Dulvy et al., 2014; Oliver et al., 2015; Dulvy \&

51 Trebilco, 2018). These species are especially vulnerable to overfishing due to life history traits

52 such as late maturity, slow growth, low fecundity, and high juvenile mortality, which collectively

53 result in low intrinsic rate of population increase (Baum et al., 2003; Dulvy et al., 2008).

54 Elasmobranch populations take decades to recover from overfishing, and only if fishing pressure

55 is relieved for an extended period (Stevens et al., 2000). Furthermore, many threatened and

56 endangered elasmobranchs have little to no population genetic data that would assist in the

57 resolution of management units (reviewed in Domingues et al., 2018a).

Genetically distinct populations are isolated management units known as stocks; however, stocks can be defined on a smaller scale than genetic populations through other criteria, such as an exclusive economic zone boundry (Carvalho \& Hauser, 1994; Ovenden et al., 2015). Reduced gene flow indicates that if a population is overfished it will not be replenished by immigrants

63

66 from surrounding populations. This is why managing on a genetic stock-by-stock basis is

essential for successful maintenance of exploited species and is sorely needed for over-harvested elasmobranchs (Dizon et al., 1993; Heist, 2004; Tallmon et al., 2010).
For the past two decades the standard for examining population structure in elasmobranchs has been a section of the mitochondrial genome, usually the control region (mtCR) (e.g. Duncan et al. 2006; Hoelzel et al. 2006; Keeney \& Heist 2006; Castro et al. 2007; Whitney et al. 2012; Clarke et al. 2015; reviewed in Domingues et al. 2018a). Though recent studies are moving 
71 towards multi-marker approaches (Momigliano et al., 2017; Pazmiño et al., 2018: Green et al.,

72 2019), there is still a large body of literature focusing on mtCR. The mitochondrial genome has a

73 higher rate of mutation than most of the nuclear genome (Brown et al., 1979; Charlesworth \&

74 Wright, 2001; Neiman \& Taylor, 2009) and this rate of mutation is a key advantage in

75 vertebrates with slowly-evolving genomes (Avise et al., 1992; Martin et al., 1992).

76 Elasmobranch mtDNA studies to date have been successful in elucidating population partitions

77 and evolutionary divergences, but the maternal inheritance of mtDNA can limit conclusions

78 about gene flow in cases of sex-biased (usually male) dispersal. Both mtDNA and nuclear

79 markers often have concordant results in sedentary species (e.g., Lavery et al., 1996; Avise,

80 2004; Zink \& Barrowclough, 2008; DiBattista et al., 2015) but, when examined alone, may miss

81 key components of population structure, particularly in migratory fauna (Pardini et al., 2001;

82 Bowen et al., 2005; Toews \& Brelsford, 2012). When highly mobile elasmobranchs are

83 examined with both mtDNA and nuclear markers (usually microsatellites), a different picture

84 often emerges in which females are more resident and males are dispersive (Pardini et al., 2001;

85 Schultz et al., 2008; Portnoy, et al., 2010; Karl, Castro, Lopez, Charvet, \& Burgess, 2011; Daly-

86 Engel et al., 2012; Portnoy et al., 2015: Bernard et al., 2017; Domingues et al., 2018b).

87 Identifying outlier SNPs in the nuclear genome can highlight genes possibly under selection, or

88 show functional responses to environmental changes that have important management

89 consequences (Barrio et al., 2016; Fischer et al., 2013; Guo et al., 2016; Jones et al., 2012).

90 Therefore, the combination of mitochondrial and nuclear markers can yield fundamental

91 ecological and evolutionary insights.

92 
93 High-throughput sequencing is a powerful tool for revealing fine-scale population structure that

94 may be missed by single locus studies (Andrews et al., 2016; Hohenlohe et al., 2018). However,

95 this method can be costly, especially when examining many individuals as is typical of

96 population genetic or phylogeography studies, and the perceived cost may prevent some from

97 considering a high-throughput sequencing approach. For population genetics approaches based

98 on differences in allele frequencies among populations, equimolar pooling of samples before

99 sequencing is an affordable and accurate strategy for large-scale genetic analysis (Schlötterer et

100 al., 2014). Several studies have successfully resolved population structure using a pooled site-

101 associated DNA approach known as pool-seq, including some in commercially valuable marine

102 species (e.g. Gautier et al., 2013; Mimee et al., 2015). Pool-seq provides estimates of allele

103 frequencies for thousands of loci distributed across the genome simultaneously, which in some

104 cases gives greater statistical power that can actually exceed the accuracy of allele frequency

105 estimates based on individual sequencing (Futschik \& Schlötterer, 2010, but also see Anderson

106 et al. 2014). Therefore, a comparison of results between the standard mtCR analysis and high-

107 throughput pool-seq is informative in evaluating the relative power and cost of the two

108 approaches for examining population structure.

109

110 The silky shark (Carcharhinus falciformis (Müller \& Henle, 1839)) is the second most

111 commonly harvested shark on Earth (Oliver et al., 2015; Rice \& Harley, 2013). They are one of

112 the top contributors to the shark fin trade and the most common elasmobranch bycatch species in

113 tuna purse-seine fisheries around the world (Cardenosa et al., 2018; Clarke et al., 2006; Oliver et

114 al., 2015). This pelagic shark, formerly abundant in all tropical oceans, has declined by an

115 estimated $85 \%$ in the last 20 years, and is now listed as vulnerable and declining by the 
116 International Union for the Conservation of Nature (Rice \& Harley, 2013; IUCN, 2017).

117 Currently silky shark population assessments are conducted at the scale of regional fishery

118 management organization, and conservation management measures are implemented at this scale

119 in the absence of genetic or movement data to define population boundaries. Clarke et al. (2015)

120 surveyed silky sharks across these regional management regions and found the western Atlantic

121 was strongly differentiated from the Indian Ocean, but the North Atlantic, Gulf of Mexico, and

122 Brazil could not be differentiated and appeared to comprise a single population. In contrast,

123 using the same mtCR marker, Domingues et al. (2017) examined five regions across the Western

124 Atlantic and found the North Western Atlantic was distinct from the South Western Atlantic. The

125 difference between the two studies results from additional sampling in the South West Atlantic

126 from further south than Clarke et al. (2015).

127

128 In an era where wildlife management needs far exceed the financial resources to address them,

129 many seek to find the most accessible, robust, and economical means to define management

130 units. In this study, we provide a direct comparison of population genetic analysis methods

131 between Sanger sequencing of the mtCR region and high-throughput sequencing of regional

132 pools of individuals. The same individuals from Clarke et al. (2015) were re-sequenced using

133 pool-seq approaches. Regions re-sequenced included Gulf of Mexico, North West Atlantic, and

134 Brazil, as well as one geographically distant location in the Red Sea (Fig 1$)$. We focused this

135 analysis on SNPs from the mitochondrial DNA as well as nuclear DNA. We did not analyze any

136 microsatellite loci because they were not a part of Clarke et al. (2015). We then evaluate the

137 economics of conducting pool-seq relative to conventional Sanger sequencing of these same 
138 individuals. Ecological and management implications will be addressed in a subsequent

139 companion paper.

140

\section{Materials \& Methods}

\section{Sampling and sequencing}

143 A total of 143 silky shark fin clips or muscle sections were sampled from commercial or artisanal

144 fisheries across four geographic regions and are the same samples examined in Clarke et al.

145 (2015). Specifically, we sampled the Gulf of Mexico (GM, n =39), the North Atlantic (NA, $n=$

146 33), Brazil (BR, $n=34)$, and the Red Sea (RS, $n=37)$. These sample sizes are slightly lower

147 than Clarke et al. (2015). This reduction was due to DNA degradation over time and the need for

148 high-quality genomic DNA for pool-seq. This is contrary to the DNA quality needed for

149 amplifying a single marker from the mitochondrial control region. Additionally only a subset of

150 the Red Sea samples were randomly selected to keep sample sizes relatively similar.

152 DNA was extracted using Qiagen DNeasy Blood \& Tissue kit (Qiagen, Mississauga, ON,

153 Canada), following manufacturer protocols. Extracted DNA quality was assessed visually by gel

154 electrophoresis and imaged using Gel Doc E-Z System (BIO RAD, Hercules, California, USA).

155 Only DNA aliquots with strong genomic DNA bands were further processed, while degraded or

156 overly digested DNA was discarded. Aliquots of high-quality DNA were quantified using an

157 AccuClear Ultra high sensitivity dsDNA quantitation kit (Biotium, Fremont CA, USA) and a

158 SpectroMax M2 (Molecular Devices, Sunnyvale, CA, USA). Libraries were pooled with an

159 equal amount of DNA ( $\mathrm{ng} / \mu \mathrm{l})$ contributed per individual to minimize individual contribution

160 bias, totaling $2000 \mathrm{ng}$ of DNA per library. Number of individuals per pool are displayed in Fig 1. 
161 No PCR was performed to ensure individual DNA contribution was kept equal within and across

162 libraries (Anderson et al., 2014). The rest of the library preparation followed the ezRAD library

163 preparation protocol (Toonen et al. 2013; Knapp et al., 2016). This included DNA digested with

164 DpnII restriction enzyme and adapters ligated using a Kapa hyper Prep Kit (Kapa Biosystems,

165 Wilmington, MA, USA). Pooled libraries were sequenced using Illumina MiSeq (v3 2x300bp

166 PE) at the Hawai' $i$ Institute of Marine Biology EPSCoR Core sequencing facility.

\section{Genetic analyses}

168 MultiQC was used to assess sequence quality scores, sequence length distributions, duplication

169 levels, and overrepresented sequences (Ewels et al., 2016). To analyze the mitochondrial

170 genome, a previously published mitochondrial genome from Carcharhinus falciformis was used

171 as a reference (GeneBank accession number KF801102). Raw paired-end reads were trimmed

172 with TRIMMOMATIC, mapped to the mitochondrial genome reference BWA (mem algorithm),

173 and variants called using the dDocent bioinformatics pipeline, modified for pool-seq (Puritz et al.

1742014 , see below for details). Called SNPs were then analyzed with AssessPool

175 (github.com/ToBoDev/assessPool, see below for details).

176

177 The bioinformatics pipeline included dDocent followed by AssessPool. Given that no reference

178 genome was available, a reference was constructed using the dDocent de novo assembly and

179 optimized utilizing the reference optimization steps provided on the dDocent assembly tutorial

180 (http://ddocent.com/assembly/). Before assembly reads were trimmed using default settings and

181 then an overlap (OL) assembly was performed, followed by clustering with CD-HIT with a -c

182 parameter of $90 \%$ similarity. For mapping using BWA (mem algorithm) all match, mismatch,

183 and gap open penalty score parameters were also default settings. Different parameters were 
184 tested during optimization but did not improve mapping. Within-pool (K1) and between-pool

185 (K2) minimum locus depth values selected for the de novo assembly did impact the results.

186 dDocent provides graphical outputs to help select these values; however, testing a few different

187 values of each is recommended to fully explore the potential of the data by balancing number of

188 contigs by coverage depth (see ddocent.com/UserGuide for details). Selected values for K1 and

189 K2 were 3 and 3 respectively. Once assembled, sequences were mapped, SNPs were called

190 within the dDocent pipeline using FreeBayes, modified for SNP calling in pools (Garrison and

191 Marth 2012, https://github.com/ekg/freebayes). Any contigs that aligned to the mitochondrial

192 genome were removed from this nuclear dataset. The contigs that aligned specifically to the

193 mitochondrial control region were saved for SNP validation to directly compare the results from

194 this pool-seq approach to those previously reported by Clarke et al. (2015).

196 SNP calling with FreeBayes was optimized for pooled samples using the 'pooled-continuous'

197 option, and minor allele frequency was decreased to 0.05 to capture alleles with frequency

198 greater than 5\% in the population (See Supplementary Material for code). The dDocent pipeline

199 outputs SNPs in two variant call format files (.vcf), one being all raw SNPs (TotalRawSNPs.vcf)

200 and another with filtered SNPs (Final.recode.vcf) however dDocent does not optimize filtering

201 for pool-seq data. Therefore, the raw SNPs were processed with the pool-seq specific program

202 AssessPool which uses VCFtools and veflib to filter SNPs (Danecek et al., 2011). SNPs were

203 processed with the following filters: minimum pool number of 2, minimum quality score of 20,

204 minimum depth threshold of 30, maximum amount of missing data of 3 , maximum allele length

205 of 10 , quality score to depth ratio of 0.25 as well as mean depth per site vs. quality score, and

206 finally a maximum mean depth threshold of 1000 (Table S1). AssessPool then sends filtered 
207 SNPs to either PoPoolation2 (Kofler et al., 2011) or poolfstat (Hivert et al., 2018). PoPoolation2

208 calculates mean pairwise $F_{S T}$ values and significance in the form of p-values obtained using

209 Fisher's exact test and combined using Fisher's method (as described in Ryman et al. 2006).

210 Poolfstat (Hivert et al. 2018) takes a different approach, calculating $F_{S T}$ values based on an

211 analysis-of-variance framework (sensu Wier \& Cockerham 1984) to eliminate biases associated

212 with varying pool sizes. AssessPool then organizes, summarizes, and creates visualizations of the

213 data using RStudio (RStudio Team 2020).

215 As a quality control test, sequences from Clarke et al. (2015) were downloaded from GenBank 216 (accession numbers KM267565-KM267626), and SNPs from these data were compared directly

217 to SNPs called within the control region of the mitochondrial pool-seq data generated here.

218 Concordance of this validation set of SNPs was determined by Mantel test in R (Legendre \&

219 Legendre, 1998) comparing the matrices of pairwise $F_{S T}$ values among populations.

\section{Cost Analysis}

221 The cost of pool-seq approach compared to Sanger sequencing of individual loci was calculated

222 based on library preparation and sequencing cost at our facility. We did not include labor but

223 calculated the total cost to generate sequence data from each sample included here from such

224 expenses as the extraction, laboratory consumables, PCR amplification, library preparation,

225 reaction clean-ups, quantification, quality control testing, and sequencing costs. These costs were

226 translated into functions in RStudio (RStudio Team, 2020) where Sanger sequencing is a fixed

227 rate per individual and pool-seq costs are fixed per flow cell on our MiSeq, but individual cost

228 varies based on number of individuals and number of pooled regions per sequencing run. These

229 functions were then plotted together for comparison. 


\section{Results}

232 A total of 30.8 million reads were generated for the four geographic regions, which averaged 7.7

$233 \pm 3.0$ million reads per pooled library. Results from the MutliQC assessment showed fairly

234 homogenous output between libraries in regard to sequence quality scores, GC and per base

235 sequence content, sequence length distributions, duplication levels, overrepresented sequences,

236 and adapter content. Once assembled, aligned, and mapped, 5,792 SNPs were resolved across the

237 mitochondrial and nuclear genomes combined. There were 4,103 biallelic SNPs, 168 were

238 multialleleic SNPs and 48 were insertions and deletions (INDELs). INDELs and multiallelic

239 SNPs remain a challenge for quantification software, so we restricted our analysis to biallelic

240 loci (Fracassetti et al. 2015). AssessPool creates visualizations of $F_{S T}$ values and allows for visual

241 outlier inspection. No visual outliers were present and given these SNPs are distributed

242 haphazardly across the genome, they are assumed to be putatively neutral.

\section{Mitochondrial Genome}

245 Analysis of the complete mitochondrial genome (17,774 bp) revealed 804 variable sites: 681

246 biallelic and 17 multiallelic SNPs. Because coverage in this dataset was fairly low on average,

247 most of these SNPs did not meet the filter threshold. After further filtering for the highest quality

248 markers, 30 SNPs were selected to calculate allele frequencies. Pairwise $F_{S T}$ values were all

249 significant (Fig 2, Table S2). The Red Sea had much higher $F_{S T}$ values (ranging from 0.367 to

250 0.745) than any inter-Atlantic comparison (ranging from 0.029 to 0.135 ). However, all

251 comparisons within the Atlantic still showed significant $F_{S T}$ values, the highest being between 
252 the North Atlantic and Brazil, and the lowest between Brazil and Gulf of Mexico (Fig 2, Table 253 S2).

\section{Nuclear loci}

256 Our nuclear data showed 4,988 variants of which 3,422 were biallelic SNPs and 151 were

257 multialleleic SNPs. A total of 346 SNPs remained after the same filtering process for the highest 258 quality SNPs was applied as for the mitochondrial genome. Nuclear markers showed lower $F_{S T}$ 259 values between locations than the mitochondrial data, yet all P-values were still significant (Fig

260 2, Table S2). The Red Sea showed consistently higher $F_{S T}$ values in comparison to inter-Atlantic 261 comparisons except for the North Atlantic to Gulf of Mexico comparison, which showed the 262 second highest mean $F_{S T}$ value (Fig 2 , Table $\left.S 2\right)$. The highest value $\left(F_{S T}=0.035\right)$ was observed 263 between Gulf of Mexico and the Red Sea, whereas the lowest $\left(F_{S T}=0.014\right)$ was between the 264 North Atlantic and Brazil, which had the highest $F_{S T}$ value within the Atlantic for the 265 mitochondrial data.

\section{SNP validation}

268 SNPs called in the mitochondrial control region using the pool-seq protocol were compared with 269 those reported in Clarke et al. (2015). Of the 34 SNPs in their study 14 of them had a minor 270 allele count (MAC) of less than or equal to 3 and several were singletons. These SNPs are 271 removed from the pool-seq data due to MAC SNP filter of $>3$ to remove sequencing errors that 272 might be scored as rare alleles during high-throughput sequencing. Therefore, singletons or any 273 rare allele represented fewer than 3 times in a population will inherently be removed from pool274 seq data sets. Fortunately those rare alleles do not tend to overly impact Fst values and should 
275 not bias interpretations of population structure (Bird et al. 2011; Toonen et al. 2011). Three SNPs

276 were found in the Clarke study with a MAC of $>3$ that were not present in the pool-seq data;

277 however, the remining 17 SNPs were all present in our data, plus one that was not found in the

278 Clarke study (Fig S1). Despite the loss of these rare alleles from the SNP validation set, pairwise

$279 F_{S T}$ values estimated by both methods remained highly correlated (Mantel test, $\mathrm{r}^{2}=0.96, \mathrm{p}<$

280 0.05), and comparisons between the Red Sea and all three Atlantic populations showed the same

281 relative magnitude between both methods.

\section{Cost Analysis}

283 The findings for cost analysis indicate that pool-seq reaches a threshold at approximately 300

284 individuals, after which this approach offers cheaper results than individual Sanger sequences.

285 Furthermore, the cost is only twice as expensive at just over 100 individuals (Fig $3 a$ ). The pool-

286 seq approach provides a far higher ratio of information for the cost, yielding greater population

287 resolution. This cost assessment does not include analytical time, labor, or effort associated with

288 pool-seq analyses such as access to computer resources and expertise with bioinformatic

289 pipelines. However these costs are likely to decrease in the near future as bioinformatic pipelines

290 are improved and become more widely available, for example as applications deployed via cloud

291 based platforms such as Galaxy (https://usegalaxy.org/) or CyVerse (https://cyverse.org/). It is

292 also important to note that the choice of pool-seq methodology has many caveats, which are

293 discussed in greater detail in the 'considerations on pool-seq' section of the discussion below.

\section{Discussion}

295 Elasmobranchs are being harvested at unsustainable levels in several commercial fishing

296 industries around the world. A fundamental step in successful management of any species is 
297 resolving population boundaries so they can be managed on a genetic stock by stock basis. As

298 genetic sequencing technologies advance, there is greater opportunity to detect even small-scale

299 genetic differences between populations. When these differences amount to statically significant

300 allele frequencies at the population level, this indicates limited exchange among distinct stocks.

301

302 Here, we validate the utility of pool-seq using the same individuals as a previous study (Clarke et 303 al. 2015) and show that pool-seq recovers additional population structure relative to Sanger

304 sequencing of the mtDNA control region. Pool-seq was able to detect isolated populations

305 between the Gulf of Mexico, Western Atlantic, and along the Brazilian coast, where Clarke et al.

306 (2015) found no population structure. As expected, the Red Sea population was highly isolated

307 from Atlantic conspecifics using both approaches.

308

309 One advantage of this pool-seq approach is that we recover SNPs throughout the entire

310 mitochondrial genome along with thousands of additional nuclear loci that together provide

311 greater statistical power to detect finer scale population structure (Ryman \& Palm 2006; Larsson

312 et al. 2009; Kurland et al. 2019). The pool-seq approach yielded significant genetic structure

313 among inter-Atlantic regions in both mtDNA and nuclear loci, whereas Sanger sequencing of the

$314 \mathrm{mtCR}$ lacked power to resolve significant differences among the same populations. The

315 congruence between the mitochondrial genome and nuclear loci reinforces the conclusion of

316 population structure among all regions sampled in this study.

317

318 In this case, pool-seq lived up to the promise of increased power to detect fine-scale structure,

319 but does it live up to the promise (Ferretti et al., 2013; Schlötterer et al., 2014) of being cost- 
320 effective? Individual extraction costs remain fixed across both approaches and Sanger

321 sequencing generally has a flat rate per individual, including PCR primers and reagents, and

322 sequencing per individual per locus. In contrast, pool-seq has a flat sequencing cost determined

323 by the number of reads generated from the high-throughput sequencing platform, plus a small

324 additional cost per pool for the exact quantification of DNA for equimolar pooling and the

325 library preparation for high-throughput sequencing. Comparing costs at our institution between a

326 single Sanger sequencing marker and pool-seq on the Illumina MiSeq platform indicates pool-

327 seq becomes less expensive when sample size of the study rises above 300 individuals. Although

328 the cost per pool is essentially fixed, when higher numbers of individuals are included per pool,

329 the price per individual analyzed is further reduced (Fig $3 b$ ). Our comparison here is limited to

33012 pools due to the maximum number of reads per lane produced on the MiSeq platform.

331 Therefore, analyzing more than 12 pools would require additional sequencing runs and result in a

332 step increase in the cost per individual/pool, although this would differ among other Illumina

333 machines (such as the HiSeq, NextSeq or NovaSeq) or other high-throughput sequencing

334 platforms (such as the PacBio Sequel II). Larger numbers of pools could be run on some of these

335 machines, but with differing individual read lengths and sequencing depths, which also bring

336 other trade-offs. Likewise, samples can also be run with individual barcodes, therefore gaining

337 the individual information lost by pooling specimens, but with increased initial setup and

338 sequencing costs. There are so many options by which to apply these methods that we cannot

339 possibly consider them all here, and the availability, cost, and trade-offs associated with each

340 should be ideally considered by individuals when designing high-throughput sequencing

341 projects. In our case, we considered only the options currently available to us through our

342 campus sequencing core, and all these pool-seq price comparisons are to a single Sanger- 
343 sequenced marker. Thus, when considering the information acquired from pool-seq compared to

344 the cost from traditional single mitochondrial marker the price per individual advantage is

345 massively amplified.

346

347 Considerations with pool-seq

348 As with any sequencing technique, there are still several factors to consider before deciding if

349 pool-seq is appropriate for a particular study. Multiple reviews have been published on high-

350 throughput and pool-seq approaches demonstrating pros, cons, and considerations with these

351 methods, which are beyond the scope of this study. Interested readers should consult Perez-

352 Enciso \& Ferretti (2010), Futschik \& Schlötterer (2010), Kofler et al. (2012), Ferretti et al.

353 (2013), Schlötterer et al., 2014, Andrews \& Luikart (2014), Andrews et al. (2016), and Kurland

354 et al. (2019).

355

356 Pooling assumes individuals are from the interbreeding individuals within a single population of

357 the same species. Therefore, care needs to be taken to avoid cryptic species, combining multiple

358 populations (Wahlund effect), or other unintentional bias when selecting individuals to pool

359 (Garnier-Géré \& Chikhi 2013). For wide ranging pelagic species such as the blue shark or

360 oceanic whitetip it seems reasonable to pool individuals from a larger area than it would be for

361 small benthic species such as horn sharks, wobbegongs, or most rays. Population structure may

362 be obscured if the geographic range per pool is too large or if there is complex population

363 structure (sensu Bowen et al. 2005), because individuals from multiple sub-populations will be

364 mixed into a single pool from which allele frequencies are calculated. Certainly pool-seq is not

365 appropriate in all cases. It is a cost-saving approach for analyses based on allele frequencies 
366 only, because individual information is lost by pooling, including haplotypes/genotypes and

367 linkage disequilibrium information. Also, pooling makes it difficult to distinguish between low

368 frequency alleles in the population and sequencing error. Therefore, careful filtering must be

369 applied to ensure only valid SNPs are analyzed instead of analyzing sequencing noise (Anand et

370 al., 2016; Schlötterer et al., 2014). Finally, the estimation of $F_{S T}$ from pooled data remains a

371 subject of some debate, and new approaches and bias corrections are being actively developed

372 (Kofler et al. 2011; Hivert et al. 2018). To account for this uncertainty, we include analyses

373 based on both the original PoPoolation2 (Kofler et al., 2011) package and the newer poolfstat

374 (Hivert et al., 2018) that explicitly considers potential biases associated with varying pool sizes.

375 The two approaches yield slightly different $F_{S T}$ values (see Table $S 2$ ), however a comparison of

376 the two $F_{S T}$ matrices shows strong correlation (Mantel $\mathrm{r}=0.991$ for mitochondrial and $\mathrm{r}=0.978$ for

377 nuclear data, $\mathrm{p}<0.05)$. Therefore, only those $F_{S T}$ values calculated by PoPoolation2 are reported

378 in the main text for ease of presentation.

379

380 Though pool-seq has been shown to be an affordable and reliable tool for population genomics

381 (Futschik \& Schlötterer, 2010; Gautier et al., 2013; Rellstab et al. 2013; Konczal et al. 2014;

382 Schlötterer et al. 2014; Kurland et al. 2019), projects with larger budgets could allocate funds for 383 any of a variety of other genomic sequencing techniques such as individual RADseq libraries

384 (Hohenlohe et al. 2010), GBS (Narum et al. 2013), SNP arrays (Qi et al. 2017), bait capture

385 (Feutry et al. 2020), or low coverage genomewide sequencing (Therkildsen \& Palumbi 2017).

386 These approaches allow for individual genotyping to examine questions that require individual-

387 level information and could provide a deeper assessment of populations. However it is also

388 important to consider not all labs can afford to generate genomic level data, especially in 
389 developing countries, and having a cost-effective alternative to single marker studies will

390 continue to be invaluable to many.

391

\section{Conclusions}

393 The finding of population structure on the scale of North Atlantic/Gulf of Mexico/Brazil is

394 nearly unprecedented for a pelagic shark. Population structure in globally distributed sharks is

395 typically detected on a scale of ocean basins (Atlantic versus Indo-Pacific, Castro et al. 2007;

396 Graves \& McDowell, 2015) and a few pelagic fishes have no population structure on a global

397 scale (e.g. Basking shark, Cetorhinus maximus, Hoelzel et al. 2006; Blue shark Prionace glauca,

398 Veríssimo et al. 2017; Wahoo, Acanthocybium solandri, Theisen et al. 2008). The resolution of

399 isolated populations on the scale of North Atlantic Ocean is more typical of coastal species than

400 pelagic species. The silky shark seems to be a pelagic species with a somewhat coastal

401 population structure. This has strong implications for international management because smaller

402 stocks imply smaller populations which are more readily depleted. At a minimum, these data

403 require rethinking a single population management approach for the Atlantic, and this pattern

404 needs to be investigated for this species across the Indo-Pacific as well.

405

406 Overall this study demonstrates pool-seq is a powerful and cost-effective tool for analyzing large

407 portions of the genome which the methods traditionally used for elasmobranchs could not

408 supply. Sharks and rays are an imperiled group of species that could benefit from advanced

409 genomic studies to outline appropriate management units. Finally, although the technology is

410 becoming cheaper and easier to apply, it is a common pitfall to assume everyone in the field can

411 afford, or must use, these approaches to produce defensible science. Bowen et al. (2014)

412 advocate judicious rather than wholesale application of genomic approaches as the most robust 
413 course of study, particularly when considering the global inequities in available research budgets.

414 Sanger sequencing is still more cost effective for small numbers of individuals, but as the

415 number of individuals included in a study rise, the cost per individual reaches the point where

416 high throughput sequencing studies can be cheaper than sequencing a single mitochondrial

417 marker from each individual. We provide an example of just such a case here, and highlight the

418 potential advantage of cost savings together with increased power for resolution of fine scale

419 population structure. Though there is still additional cost of using cluster computer servers and

420 bioinformatics programs, these cost are dropping as technology advances. When study organism

421 and sampling strategies are assessed and implemented into the study design, pool-seq has great

422 promise for augmenting the scientific foundations for management of marine recourses.

423

424 Acknowledgements

425 This study was made possible by the generous donation of specimens by Christopher R. Clarke,

426 Mahmood Shivji, Stephen A. Karl, J.D. Filmalter, and Julia Spaet. We thank members of the

427 ToBo Lab for sharing expertise, advice and discussions that contributed to this manuscript.

428 Special thanks to Darren Lerner, Kim Holland, Carl Meyer, S. Gulak, D. Bethe, D. McCauley, C.

429 Wilson, Guy Harvey Ocean Foundation, and Save Our Seas Foundation. This paper is funded in

430 part by a cooperative agreement from the National Oceanic and Atmospheric Administration,

431 Project R/SS-19PD, which is sponsored by the University of Hawai'i Sea Grant College Program

432 under Institutional Grant No. NA14OAR4170071 (B.W.B) from NOAA Office of Sea Grant,

433 Department of Commerce. The views expressed herein are those of the authors and do not

434 necessarily reflect the views of NOAA or any of its subagencies. This is contribution \#1821 from

435 the Hawaii Institute of Marine Biology, contribution \#XXXX from the Hawaii Sea Grant 
436 Program, and contribution \#11128 from the School of Ocean and Earth Science and Technology

437 at the University of Hawaii.

438 Author Contributions:

439 Derek W. Kraft - Designed research, performed research, analyzed data, wrote paper, provided 440 funding for research.

441 Emily Conklin - Created bioinformatics pipeline for data analysis and assisted in data analysis

442 Evan Barba - Created bioinformatics pipeline for data analysis and assisted in data analysis

443 Melanie Hutchinson - Helped obtain samples and provided biological insight

444 Robert J. Toonen - Contributed funding, contributed to research design, and contributed to data 445 analysis and edited paper

446 Zac H. Forsman - Contributed to research design and data analysis

447 Brian W. Bowen - Contributed funding, contributed to research design, and edited paper 448

\section{References}

450

451 Anand, S., Mangano, E., Barizzone, N., Bordoni, R., Sorosina, M., Clarelli, F., Corrado, L., 452 Boneschi, F. M., D’Alfonso, S., \& De Bellis, G. (2016). Next generation sequencing of 453 pooled samples: Guideline for variants' filtering. Scientific Reports, 6(August), 1-9. $454 \quad$ https://doi.org/10.1038/srep33735

455 Anderson, E. C., Skaug, H. J., \& Barshis, D. J. (2014). Next-generation sequencing for molecular 456 ecology: A caveat regarding pooled samples. In Molecular Ecology (Vol. 23, Issue 3, pp. 457 502-512). https://doi.org/10.1111/mec.12609

458 Andrews, K.R., \& Luikart, G. (2014). Recent novel approaches for population genomics data 
analysis. Molecular Ecology, 23(7), 1661-1667.

460 Andrews, Kimberly R., Good, J. M., Miller, M. R., Luikart, G., \& Hohenlohe, P. A. (2016).

461 Harnessing the power of RADseq for ecological and evolutionary genomics. Nature

462 Reviews Genetics, 17(2), 81-92. https://doi.org/10.1038/nrg.2015.28

463 Avise, J. C., Bowen, B. W., Bermingham, E., Meylan, A. B., \& Lamb, T. (1992). Mitochondrial

464 DNA evolution at a turtle's pace: evidence for low genetic variability and reduced

465 microevolutionary rate in the Testudines. Molecular Biology \& Evolution, 9, 457-473.

466

467

468

469

470

471

472

473

474

475

476

477

478

479

480

481

Barrio, A. M., Lamichhaney, S., Fan, G., Rafati, N., Pettersson, M., Zhang, H., Dainat, J., Ekman, D., Höppner, M., Jern, P., Martin, M., Nystedt, B., Liu, X., Chen, W., Liang, X., Shi, C., Fu, Y., Ma, K., Zhan, X., ... Andersson, L. (2016). The genetic basis for ecological adaptation of the Atlantic herring revealed by genome sequencing. ELife, 5(MAY2016), 132. https://doi.org/10.7554/eLife.12081

Baum, J. K., Myers, R. A., Kehler, D. G., Worm, B., Harley, S. J., \& Doherty, P. A. (2003). Collapse and conservation of shark populations in the Northwest Atlantic. Science, 299(5605), 389-392. https://doi.org/10.1126/science.1079777

Bernard, A. M., Horn, R. L., Chapman, D. D., Feldheim, K. A., Garla, R. C., Brooks, E. J., Gore, M. A., \& Shivji, M. S. (2017). Genetic connectivity of a coral reef ecosystem predator: the population genetic structure and evolutionary history of the Caribbean reef shark (Carcharhinus perezi). Journal of Biogeography, 44(11), 2488-2500. https://doi.org/10.1111/jbi.13062

Bird, C.E., Karl, S.A., Smouse, P.E. and Toonen, R.J. (2011). Detecting and measuring genetic differentiation. Phylogeography and population genetics in Crustacea, 19(3):31-55.

Bowen, B. W., Bass, A. L., Soares, L., \& Toonen, R. J. (2005). Conservation implications of 
482

483

484

485

486

487

488

489

490

491

492

493

494

495

496

497

498

499

500

501

502

503

504

complex population structure : lessons from the loggerhead turtle ( Caretta caretta ). Molecular Ecology, 14, 2389-2402. https://doi.org/10.1111/j.1365-294X.2005.02598.X

Bowen, B.W., Shanker, K., Yasuda, N., Celia, M., Malay, M.C.M.D., von der Heyden, S., Paulay, G., Rocha, L.A., Selkoe, K.A., Barber, P.H. and Williams, S.T. (2014). Phylogeography unplugged: comparative surveys in the genomic era. Bulletin of Marine Science, 90(1):13-46.

Brown, W. M., George, M., \& Wilson, A. C. (1979). Rapid evolution of animal mitochondrial DNA. Proceedings of the National Academy of Sciences of the United States of America, 76(4), 1967-1971. https://doi.org/10.1146/annurev.es.18.110187.001413

Cardeñosa, D., Fields, A. T., Babcock, E. A., Zhang, H., Feldheim, K., Shea, S. K. H., Fischer, G. A., \& Chapman, D. D. (2018). CITES-listed sharks remain among the top species in the contemporary fin trade. Conservation Letters, 11(4), 1-7. https://doi.org/10.1111/conl.12457

Carvalho, G. R., \& Hauser, L. (1994). Molecular genetics and the stock concept in fisheries. Reviews in Fish Biology and Fisheries, 4(3), 326-350. https://doi.org/10.1007/BF00042908

Castro, A. L. F., Stewart, B. S., Wilson, S. G., Hueter, R. E., Meekan, M. G., Motta, P. J., Bowen, B. W., \& Karl, S. A. (2007). Population genetic structure of Earth's largest fish, the whale shark (Rhincodon typus). Molecular Ecology, 16(24), 5183-5192. https://doi.org/10.1111/j.1365-294X.2007.03597.x

Charlesworth, D., \& Wright, S. I. (2001). Breeding systems and genome evolution. Current Opinion in Genetics \& Development, 11(6), 685-690. https://doi.org/10.1016/S0959437X(00)00254-9

Clarke, C. R., Karl, S. A., Horn, R. L., Bernard, A. M., Lea, J. S., Hazin, F. H., Prodöhl, P. A., \& 
505

506

507

508

509

510

511

512

513

514

515

516

517

518

519

520

521

522

523

524

525

526

527

Shivji, M. S. (2015). Global mitochondrial DNA phylogeography and population structure of the silky shark, Carcharhinus falciformis. Marine Biology, 162(5), 945-955. https://doi.org/10.1007/s00227-015-2636-6

Clarke, S. C., McAllister, M. K., Milner-Gulland, E. J., Kirkwood, G. P., Michielsens, C. G. J., Agnew, D. J., Pikitch, E. K., Nakano, H., \& Shivji, M. S. (2006). Global estimates of shark catches using trade records from commercial markets. Ecology Letters, 9(10), 1115-1126. https://doi.org/10.1111/j.1461-0248.2006.00968.x

Daly-Engel, T. S., Seraphin, K. D., Holland, K. N., Coffey, J. P., Nance, H. A., Toonen, R. J., \& Bowen, B. W. (2012). Global phylogeography with mixed-marker analysis reveals malemediated dispersal in the endangered scalloped hammerhead shark (sphyrna lewini). PLoS ONE, 7(1), e29986. https://doi.org/10.1371/journal.pone.0029986

Danecek, P., Auton, A., Abecasis, G., Albers, C. A., Banks, E., Depristo, M. A., Handsaker, R. E., Lunter, G., Marth, G. T., Sherry, S. T., Mcvean, G., \& Durbin, R. (2011). The variant call format and VCFtools. 27(15), 2156-2158.

https://doi.org/10.1093/bioinformatics/btr330

DiBattista, J. D., Waldrop, E., Rocha, L. A., Craig, M. T., Beruman, M. L., \& Bowen, B. W. (2015). Blinded by the bright: A lack of congruence between color morphs, phylogeography, and taxonomy in a cosmopolitan Indo-Pacific butterflyfish, Chaetodon auriga. Journal of Biogeography, 42, 1919-1929.

Dizon, A. E., Lockyer, C., Perrin, W. F., Demaster, D. P., \& Sisson, J. (1993). Rethinking the stock concept: a phylogeographic approach. Biological Conservation, 64(2), 176-177. https://doi.org/10.1016/0006-3207(93)90670-V

Domingues, R. R., Hilsdorf, A. W. S., Shivji, M. M., Hazin, F. V. H., \& Gadig, O. B. F. (2017). 
Effects of the Pleistocene on the mitochondrial population genetic structure and demographic history of the silky shark (Carcharhinus falciformis) in the western Atlantic Ocean. Reviews in Fish Biology and Fisheries, 28, 213 - 227. https://doi.org/10.1007/s11160-017-9504-z

Domingues, R. R., Hilsdorf, A. W. S., \& Gadig, O. B. F. (2018a). The importance of considering genetic diversity in shark and ray conservation policies. Conservation Genetics, 19, 501525. https://doi.org/10.1007/s10592-017-1038-3

Domingues, R. R., Bruels, C. C., Gadig, O. B. F., Chapman, D. D., Hilsdorf, A. W. S., \& Shivji, M. S. (2018b). Genetic connectivity and phylogeography of the night shark (Carcharhinus signatus) in the western Atlantic Ocean: Implications for conservation management. Aquatic Conservation: Marine and Freshwater Ecosystems, 29(1), 102-114.

Dulvy, N. K., Baum, J. K., Clarke, S., Compagno, L. J. V., Cortés, E., Domingo, A., Fordham, https://doi.org/10.1002/aqc.2961 conservation of oceanic pelagic sharks and rays. Aquatic Conservation: Marine and

Dulvy, N. K., Fowler, S. L., Musick, J. A., Cavanagh, R. D., Kyne, P. M., Harrison, L. R., Carlson, J. K., Davidson, L. N. K., Fordham, S. V., Francis, M. P., Pollock, C. M., Simpfendorfer, C. A., Burgess, G. H., Carpenter, K. E., Compagno, L. J. V., Ebert, D. A., 548 Gibson, C., Heupel, M. R., Livingstone, S. R., ... White, W. T. (2014). Extinction risk and 549 conservation of the world's sharks and rays. ELife, 2014(3), 1-34. https://doi.org/10.7554/eLife.00590.001 
551 Dulvy, N. K., \& Trebilco, R. (2018). Size-Based Insights into the Ecosystem Role of Sharks and

552 Rays. In Shark Research: Emerging Technologies and Applications for the Field and

$553 \quad$ Laboratory.

554 Duncan, K. M., Martin, A. P., Bowen, B. W., \& De Couet, H. G. (2006). Global phylogeography

555 of the scalloped hammerhead shark (Sphyrna lewini). Molecular Ecology, 15(8), 2239-

556 2251. https://doi.org/10.1111/j.1365-294X.2006.02933.x

557 Ewels, P., Lundin, S., \& Max, K. (2016). Data and text mining MultiQC : summarize analysis

558 results for multiple tools and samples in a single report. Bioinformatics, 32(19), 3047-3048.

559 https://doi.org/10.1093/bioinformatics/btw354

560 Ferretti, F., Worm, B., Britten, G. L., Heithaus, M. R., \& Lotze, H. K. (2010). Patterns and

561 ecosystem consequences of shark declines in the ocean. In Ecology Letters (Vol. 13, Issue

562 8, pp. 1055-1071). https://doi.org/10.1111/j.1461-0248.2010.01489.x

563 Ferretti, L., Ramos-Onsins, S. E., \& Pérez-Enciso, M. (2013). Population genomics from pool

564 sequencing. Molecular Ecology, 22(22), 5561-5576. https://doi.org/10.1111/mec.12522

565 Feutry, P., Devloo-Delva, F., Tran Lu Y, A., Mona, S., Gunasekera, R.M., Johnson, G., Pillans,

566 R.D., Jaccoud, D., Kilian, A., Morgan, D.L. and Saunders, T. (2020). One panel to rule

567 them all: DArTcap genotyping for population structure, historical demography, and kinship

568 analyses, and its application to a threatened shark. Molecular Ecology Resources. Online

$569 \quad$ Early, https://doi.org/10.1111/1755-0998.13204

570 Fischer, M. C., Rellstab, C., Tedder, A., Zoller, S., Gugerli, F., Shimizu, K. K., Holderegger, R.,

571 \& Widmer, A. (2013). Population genomic footprints of selection and associations with

572 climate in natural populations of Arabidopsis halleri from the Alps. Molecular Ecology,

573 22(22), 5594-5607. https://doi.org/10.1111/mec.12521 
574 Futschik, A., \& Schlötterer, C. (2010). The next generation of molecular markers from massively

575 parallel sequencing of pooled DNA samples. Genetics, 186(1), 207-218.

$576 \quad$ https://doi.org/10.1534/genetics.110.114397

577 Garnier-Géré, P., \& Chikhi, L. (2013) Population subdivision, Hardy-Weinberg equilibrium, and

578 the Wahlund effect. eLS Books, Wiley Online Library Pub. 3

579 https://onlinelibrary.wiley.com/doi/abs/10.1002/9780470015902.a0005446.pub3

580 Garrison, E., \& Marth, G. (2012). Haplotype-based variant detection from short-read sequencing. $581 \quad 1-9$

582 Gautier, M., Foucaud, J., Gharbi, K., Cézard, T., Galan, M., Loiseau, A., Thomson, M., Pudlo,

583 P., Kerdelhué, C., \& Estoup, A. (2013). Estimation of population allele frequencies from

584 next-generation sequencing data: Pool-versus individual-based genotyping. Molecular

585 Ecology, 22(14), 3766-3779. https://doi.org/10.1111/mec.12360

586 Green, M. E., Appleyard, S. A., White, W., Tracey, S., Devloo-Delva, F., \& Ovenden, J. R.

587 (2019). Novel multimarker comparisons address the genetic population structure of silvertip

588 sharks (Carcharhinus albimarginatus). Marine and Freshwater Research, 70(7), 1007-1019.

589 https://doi.org/10.1071/MF18296

590 Guo, B., Li, Z., \& Merilä, J. (2016). Population genomic evidence for adaptive differentiation in

591 the Baltic Sea herring. Molecular Ecology, 25(12), 2833-2852.

$592 \quad$ https://doi.org/10.1111/mec.13657

593 Heist, E. J. (2004). Biology of Sahrks and Their Relatives (J. C. Carrier, J. A. Musick, \& M. .

594 Heithaus (eds.)). CRC Press, Boca Raton, FL.

595 Heithaus, M. R., Frid, A., Wirsing, A. J., \& Worm, B. (2008). Predicting ecological

596 consequences of marine top predator declines. Trends in Ecology and Evolution, 23(4), 
Heupel, M. R., Knip, D. M., Simpfendorfer, C. A., \& Dulvy, N. K. (2014). Sizing up the ecological role of sharks as predators. Marine Ecology Progress Series, 495, 291-298.

$600 \quad$ https://doi.org/10.3354/meps 10597

601 Hivert, V., Leblois, R., Petit, E. J., Gautier, M., \& Vitalis, R. (2018). Measuring genetic 602 differentiation from Pool-seq data. Genetics, 210(September), 315-330.

603 https://doi.org/10.1534/genetics. 118.300900

604 Hoelzel, A. R., Shivji, M. S., Magnussen, J. E., \& Francis, M. P. (2006). Low worldwide genetic 605 diversity in the basking shark ( Cetorhinus maximus ). Biology Letters, 2, 639-642.

606 https://doi.org/10.1098/rsbl.2006.0513

607 Hohenlohe, P. A., Hand, B. K., Andrews, K. R., \& Luikart, G. (2018). Population genomics 608 provides key insights in ecology and evolution. Population Genomics: Concepts, 609 Approaches and Applications, doi:10.1007/13836_2018_20. https://doi.org/10.1007/13836_2018_20

611 Hohenlohe, P.A., Bassham, S., Etter, P.D., Stiffler, N., Johnson, E.A. and Cresko, W.A. (2010). 612 Population genomics of parallel adaptation in threespine stickleback using sequenced RAD 613 tags. PLoS Genetics 6(2): e1000862.

614 IUCN, I. U. for the C. of N. (2017). IUCN Red List of Threatened Species. Version 2013.1. 615 http://www.iucnredlist.org/about/overview

616 Jones, F. C., Grabherr, M. G., Chan, Y. F., Russell, P., Mauceli, E., Johnson, J., Swofford, R., 617 Pirun, M., Zody, M. C., White, S., Birney, E., Searle, S., Schmutz, J., Grimwood, J., 618 Dickson, M. C., Myers, R. M., Miller, C. T., Summers, B. R., Knecht, A. K., ... Kingsley, 619 D. M. (2012). The genomic basis of adaptive evolution in threespine sticklebacks. Nature, 
621 Karl, S. A., Castro, A. L. F., Lopez, J. A., Charvet, P., \& Burgess, G. H. (2011). Phylogeography 622 and conservation of the bull shark (Carcharhinus leucas) inferred from mitochondrial and 623 microsatellite DNA. Conservation Genetics, 12(2), 371-382. https://doi.org/10.1007/s10592-010-0145-1

Karl, S. a, Bowen, B. W., \& Avise, J. C. (1992). Global population genetic structure and malemediated gene flow in the green turtle (Chelonia mydas): RFLP analysis of anonymous nuclear loci. Genetics, 131, 163-173. https://doi.org/10.1139/z05-185

Keeney, D. B., \& Heist, E. J. (2006). Worldwide phylogeography of the blacktip shark (Carcharhinus limbatus) inferred from mitochondrial DNA reveals isolation of western Atlantic populations coupled with recent Pacific dispersal. Molecular Ecology, 15, 3669-

Knapp, I. S. S., Puritz, J. B., Bird, C. E., Whitney, J. L., Sudek, M., Forsman, Z. H., \& Toonen, R. J. (2016). ezRAD- an accessible next-generation RAD sequencing protocol suitable for non-model organisms_v3.2.https://doi.org/dx.doi.org/10.17504/protocols.io.e9pbh5n

Kofler, R., Betancourt, A. J., \& Schlötterer, C. (2012). Sequencing of pooled DNA samples (Pool-Seq) uncovers complex dynamics of transposable element insertions in Drosophila melanogaster. PLoS Genetics, 8(1). https://doi.org/10.1371/journal.pgen.1002487

Kofler, R., Pandey, R. V., \& Schlötterer, C. (2011). PoPoolation2: Identifying differentiation between populations using sequencing of pooled DNA samples (Pool-Seq). Bioinformatics, 27(24), 3435-3436. https://doi.org/10.1093/bioinformatics/btr589 frequency estimation using pooled RNA-Seq. Molecular Ecology Resources, 14(2):381- 
643 392.

644 Kurland, S., Wheat, C.W., de la Paz Celorio Mancera, M., Kutschera, V.E., Hill, J., Andersson, 645 A., Rubin, C.J., Andersson, L., Ryman, N. and Laikre, L., (2019). Exploring a

646 Pool-seq-only approach for gaining population genomic insights in nonmodel 647 species. Ecology and evolution, 9(19), pp.11448-11463.

648 Larsson, L.C., Charlier, J., Laikre, L. and Ryman, N.(2009). Statistical power for detecting 649 genetic divergence — organelle versus nuclear markers. Conservation Genetics, 10(5): $650 \quad 1255$.

651 Legendre, P., \& Legendre, L. (1998). Numerical Ecology. 2nd English Edition.

652 Martin, A. P., Naylor, G., \& Palumbi, S. R. (1992). Rates of mitochondrial DNA evolution in 653 sharks are slow compared to mammals. Nature, 357, 153-155.

654 https://doi.org/10.1038/357153a0

655 Mimee, B., Duceppe, M. O., Véronneau, P. Y., Lafond-Lapalme, J., Jean, M., Belzile, F., \& 656 Bélair, G. (2015). A new method for studying population genetics of cyst nematodes based 657 658 15(6), 1356-1365. https://doi.org/10.1111/1755-0998.12412

Momigliano, P., Harcourt, R., Robbins, W. D., Jaiteh, V., Mahardika, G. N., Sembiring, A., \& 660 Stow, A. (2017). Genetic structure and signatures of selection in grey reef sharks 661 (Carcharhinus amblyrhynchos). Heredity, 119(3), 142-153.

662 https://doi.org/10.1038/hdy.2017.21

663 Musick, J. A., Burgess, G., Cailliet, G., Camhi, M., \& Fordham, S. (2000). Management of 664 Sharks and Their Relatives (Elasmobranchii). Fisheries, 25(3), 9-13.

665 https://doi.org/10.1577/1548-8446(2000)025<0009:MOSATR>2.0.CO;2 
666 Myers, R. A., Baum, J. K., Shepherd, T. D., Powers, S. P., \& Peterson, C. H. (2007). Cascading 667 effects of the loss of apex predatory sharks from a coastal ocean. Science, 315(5820), 1846668 1850. https://doi.org/10.1126/science.1138657

669 Neiman, M., \& Taylor, D. R. (2009). The causes of mutation accumulation in mitochondrial 670 genomes. Proceedings of the Royal Society B: Biological Sciences, 276(1660), 1201-1209. $671 \quad$ https://doi.org/10.1098/rspb.2008.1758

672 Narum, S.R., Buerkle, C.A., Davey, J.W., Miller, M.R. and Hohenlohe, P.A. (2013).

673 Genotyping-by-sequencing in ecological and conservation genomics. Molecular 674 ecology, 22(11): 2841-2847.

675 Oliver, S., Braccini, M., Newman, S. J., \& Harvey, E. S. (2015). Global patterns in the bycatch 676 of sharks and rays. Marine Policy, 54, 86-97. https://doi.org/10.1016/j.marpol.2014.12.017

677 Ovenden, J. R., Berry, O., Welch, D. J., Buckworth, R. C., \& Dichmont, C. M. (2015). Ocean 's 678 eleven : a critical evaluation of the role of population, evolutionary and molecular genetics 679 in the management of wild fi sheries. Fish Fisheries, 16(125-159).

680 https://doi.org/10.1111/faf.12052

681 Pardini, A. T., Jones, C. S., Noble, L. R., Kreiser, B., \& Malcolm, H. (2001). Sex-biased 682 dispersal of great white sharks. Nature, 412, 139-140.

683 Pazmiño, D. A., Maes, G. E., Green, M. E., Simpfendorfer, C. A., Hoyos-Padilla, E. M., Duffy, 684 C. J. A., Meyer, C. G., Kerwath, S. E., Salinas-De-León, P., \& Van Herwerden, L. (2018). 685 Strong trans-Pacific break and local conservation units in the Galapagos shark 686 (Carcharhinus galapagensis) revealed by genome-wide cytonuclear markers. Heredity, 687 120(5), 407-421. https://doi.org/10.1038/s41437-017-0025-2

688 Perez-Enciso, M., \& Ferretti, L. (2010). Massive parallel sequencing in animal genetics : 

2052.2010.02057.x

691 Portnoy, D. S., McDowell, J. R., Heist, E. J., Musick, J. A., \& Graves, J. E. (2010). World 692 phylogeography and male-mediated gene flow in the sandbar shark, Carcharhinus 693 plumbeus. Molecular Ecology, 19, 1994-2010.

694 Portnoy, D.S., Puritz, J.B., Hollenbeck, C.M., Gelsleichter, J., Chapman, D. and Gold, J.R. 695 (2015). Selection and sex-biased dispersal in a coastal shark: The influence of philopatry on adaptive variation. Molecular Ecology, 24(23), pp.5877-5885.

697

Puritz, J. B., Hollenbeck, C. M., \& Gold, J. R. (2014). dDocent : a RADseq, variant-calling pipeline designed for population genomics of non-model organisms. PeerJ, 2, e431. https://doi.org/10.7717/peerj.431

Qi, H., Song, K., Li, C., Wang, W., Li, B., Li, L. and Zhang, G. (2017). Construction and evaluation of a high-density SNP array for the Pacific oyster (Crassostrea gigas). PLoS One, 12(3), p.e0174007.

703

704

705

706

707

708 709

Rice, J., \& Harley, S. (2013). Updated stock assessment of the Silky sharks in the Western and Central Pacfific Ocean. WCPFC-SC9-2013/SA-WP-03, August.

RStudio Team (2020). RStudio: Integrated Development Environment for R. RStudio, PBC, Boston, MA

URL http://www.rstudio.com/

Ryman, N. and Palm, S., (2006). POWSIM: a computer program for assessing statistical power when testing for genetic differentiation. Molecular Ecology Notes, 6(3), pp.600-602.

Schlötterer, C., Tobler, R., Kofler, R., \& Nolte, V. (2014). Sequencing pools of individualsmining genome-wide polymorphism data without big funding. Nature Reviews Genetics, 
713 Schultz, J. K., Feldheim, K. A., Gruber, S. H., Ashley, M. V. H., McGovern, T. M., Bowen, B.

714 W., Bruber, S. H., Ashley, M. V. H., McGovern, T. M., \& Bowen, B. W. (2008). Global

715 phylogeography and seascape genetics of the lemon sharks (genus Negaprion). Molecular

Ecology, 17(24), 5336-5348. https://doi.org/10.1111/j.1365-294X.2008.04000.x

717 Stevens, J. D., Bonfil, R., Dulvy, N. K., \& Walker, P. A. (2000). The effects of fishing on sharks, 718 rays, and chimaeras (chondrichthyans), and the implications for marine ecosystems. ICES

719 Journal of Marine Science, 57(3), 476-494. https://doi.org/10.1006/jmsc.2000.0724

720 Tallmon, D. A., Gregovich, D., Waples, R., \& Baker, C. S. (2010). When are genetic methods

721 useful for estimating contemporary abundance and detecting population trends? Molecular

722 Ecology Resources, 10, 684-692.

723 Theisen, T. C., Bowen, B. W., Lanier, W., \& Baldwin, J. D. (2008). High connectivity on a

724 global scale in the pelagic wahoo, Acanthocybium solandri (tuna family Scombridae).

725 Molecular Ecology, 17, 4233-4247.

726 Therkildsen, N.O. and Palumbi, S.R. (2017). Practical low-coverage genomewide sequencing of

727 hundreds of individually barcoded samples for population and evolutionary genomics in

728 nonmodel species. Molecular ecology resources, 17(2):194-208.

729 Toonen, R.J. and Grosberg, R.K. (2011). Causes of chaos: spatial and temporal genetic

730 heterogeneity in the intertidal anomuran crab Petrolisthes cinctipes. Phylogeography and

731 population genetics in Crustacea, 2011: 75-107.

732 Toonen, R. J., Puritz, J. B., Forsman, Z. H., Whitney, J. L., Fernandez-Silva, I., Andrews, K. R.,

733 \& Bird, C. E. (2013). ezRAD: a simplified method for genomic genotyping in non-model

734 organisms. PeerJ, 1, e203. https://doi.org/10.7717/peerj.203 
735 Weir, B.S. and Cockerham, C.C. (1984). Estimating F-statistics for the analysis of population 736 structure. Evolution 38(6):1358-1370.

737 Whitney, N. M., Robbins, W. D., Schultz, J. K., Bowen, B. W., \& Holland, K. N. (2012).

738 Phylogeography of the whitetip reef shark (Triaenodon obesus): a sedentary species with a 739 broad distribution. Journal of Biogeography, 39, 1144-1156.

740 Zink, R.M., Barrowclough, G. (2008). Mitochondrial DNA under siege in avian

741 phylogeography. Molecular Ecology, 17, 2107-2121.

742 
Figure 1

Sample locations of Carcharhinus falciformis followed by sample size

Abbreviations: $\mathrm{GM}=$ Gulf of Mexico, NA $=$ North Atlantic, $\mathrm{BR}=$ Brazil, $\mathrm{RS}=$ Red Sea .

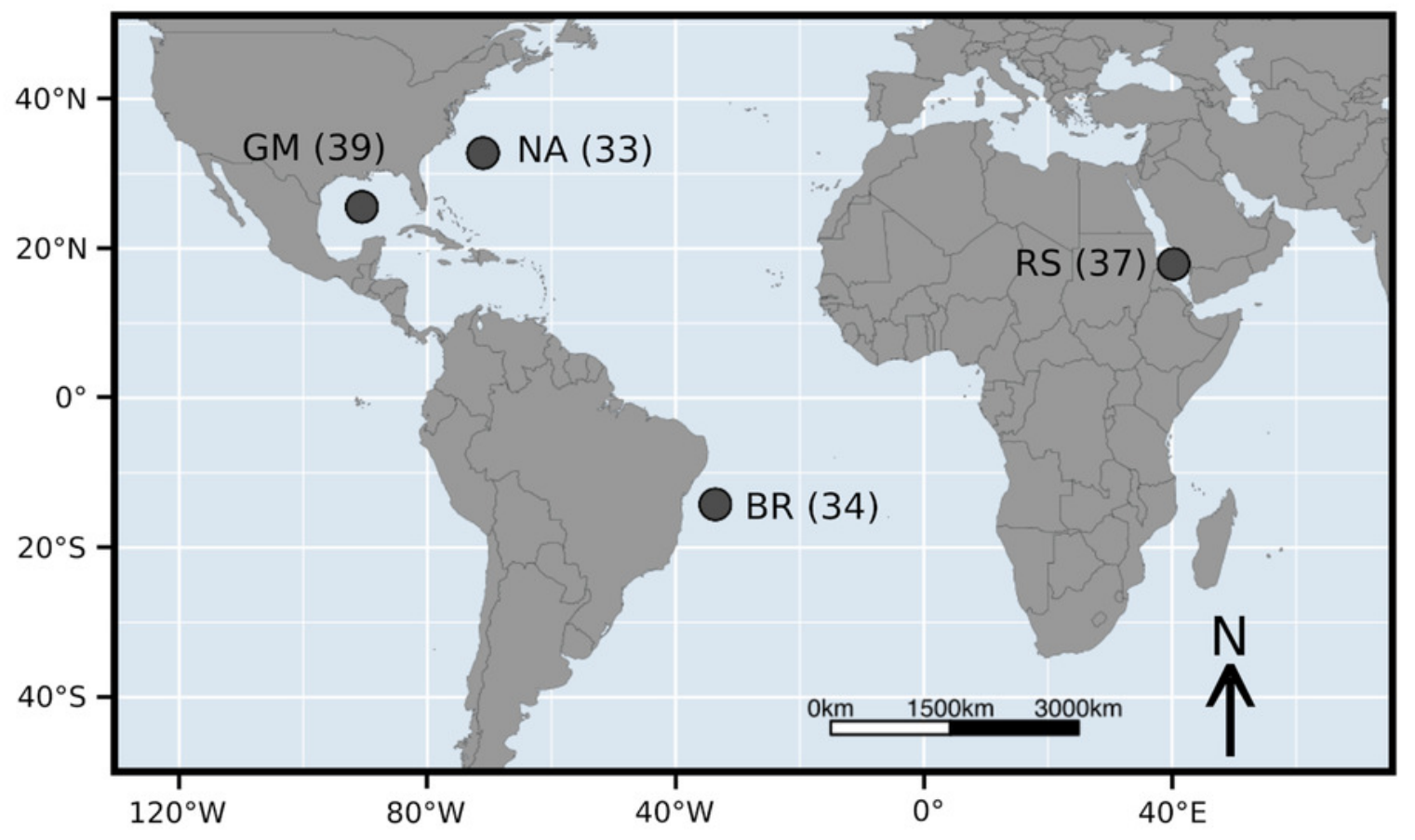




\section{Figure 2}

$\mathrm{F}_{\mathrm{st}}$ heat map for mitochondrial and nuclear data compaired to Clarke et al. Phi ${ }_{\mathrm{st}}$ and Pvalues

Figure 2. (A) Pairwise FST values generated by Pool-seq methods. Cool colors (top left) are FST values calculated from nuclear genome loci, warm colors (bottom right) are FST values from loci across the entire mitochondrial genome. All pairwise differences are significant ( $p$ $<0.001)$. (B) PhiST results from Clarke et. Al (2015) on the lower right triangle and P-values on the upper right triangle. Significant P-values and corresponding PhiST values in bold.

Regional abbreviation are as follows; $\mathrm{GM}=$ Gulf of Mexico, BR $=$ Brazil, NA $=$ North Atlantic, $\mathrm{RS}=$ Red sea. 

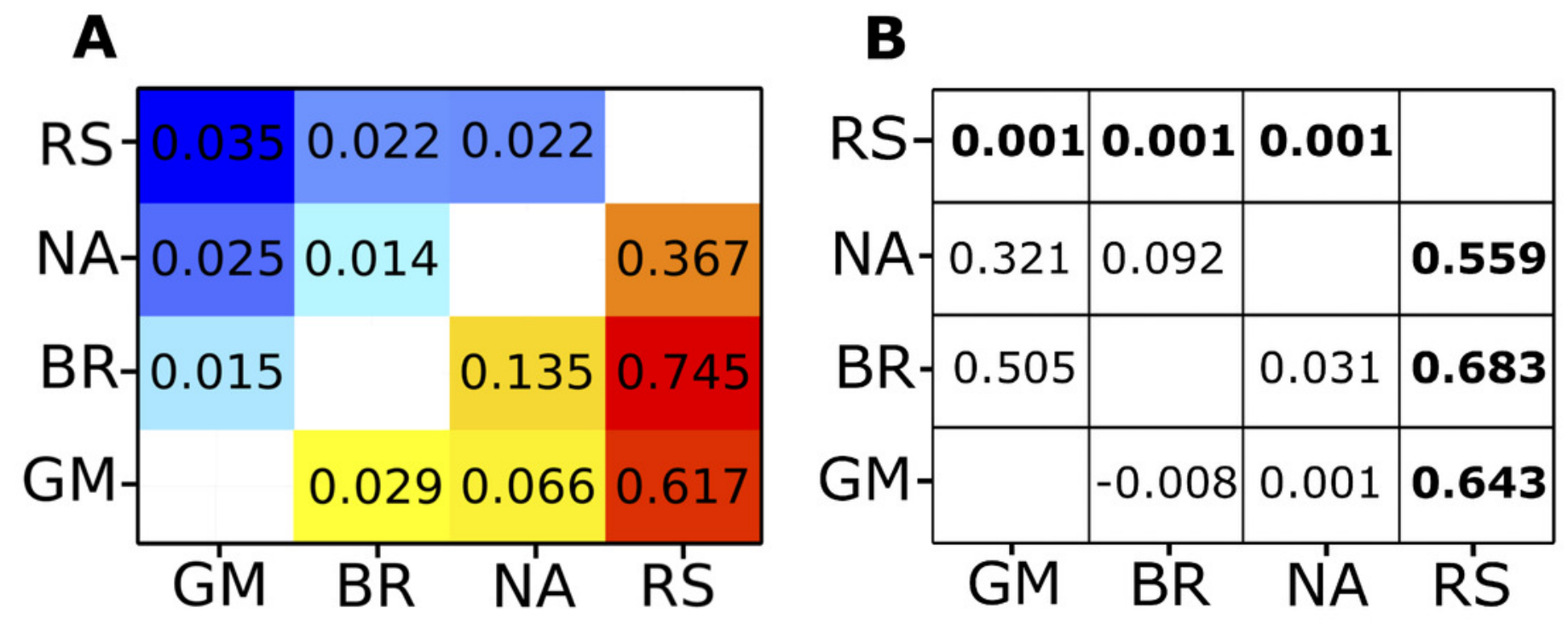
Figure 3

Cost comparisons between sequencing projects using a single Sanger marker to projects using Pool-seq with varying numbers of pools.

(A) Sequencing costs comparing number of individuals to total cost between Sanger at our facility and three Pool-seq projects at our facility containing 4, 8, and 12 pools respectively, where pool sizes change with number of individuals. (B) Sequencing cost per individual with fixed pools across different number of Pools. 

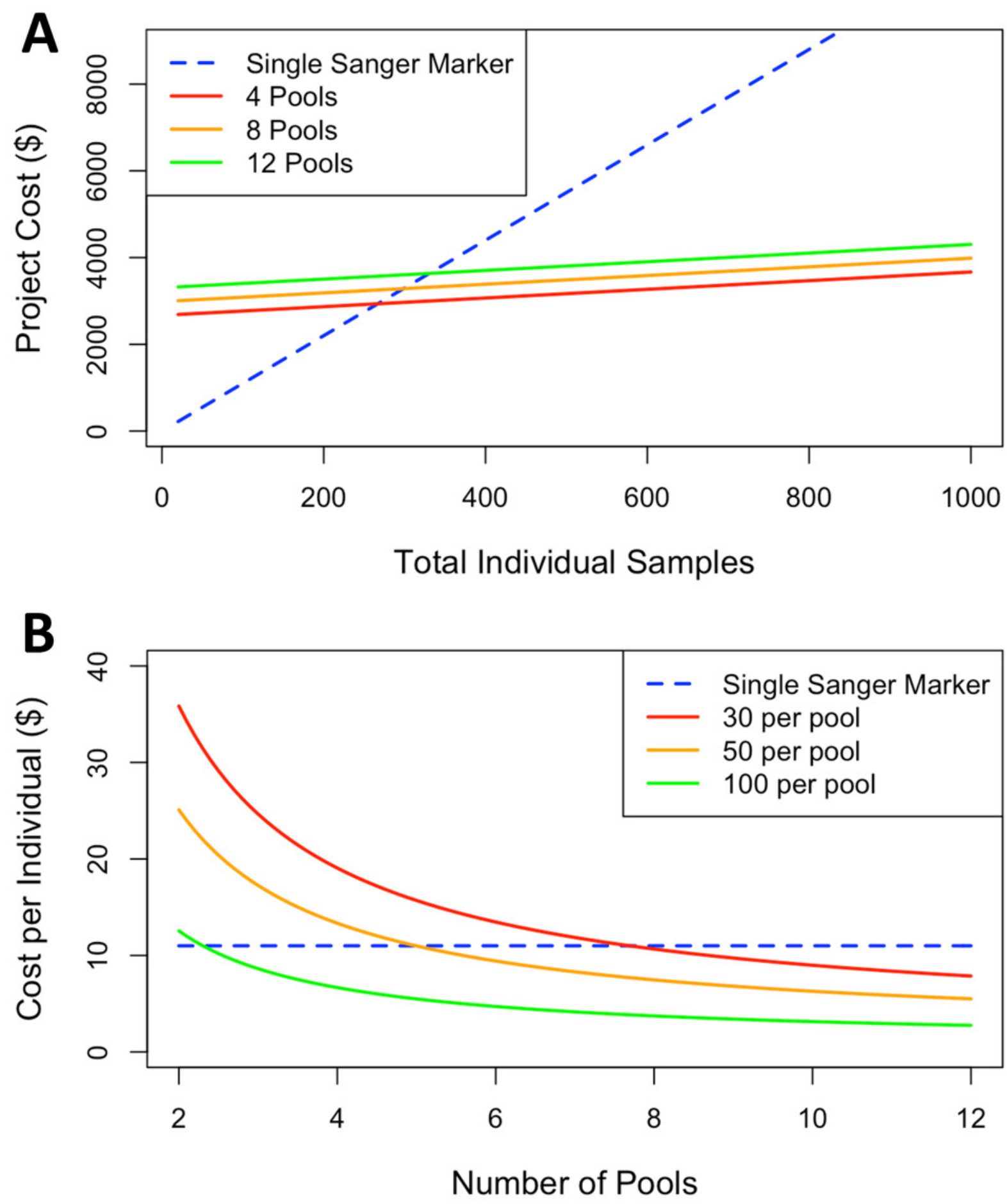